\title{
A Nanosensor Toolbox for Rapid, Label-Free Measurement of Airway Surface Liquid and Epithelial Cell Function
}

\author{
Rositsa Ivanova, ${ }^{\dagger}$ David C. H. Benton, ${ }^{\dagger}$ Mustafa M. Munye, ${ }^{\ddagger}$ Sarinda Rangseesorranan, ${ }^{\dagger}$
} Stephen L. Hart, ${ }^{+}$and Guy W. J. Moss ${ }^{*} \dagger, \S(0)$

${ }^{\dagger}$ Department of Neuroscience, Physiology and Pharmacology, ${ }^{\ddagger}$ UCL Great Ormond Street Institute of Child Health, and ${ }^{\S}$ Centre for Computation, Mathematics and Physics in the Life Sciences and Experimental Biomedicine, University College London, London WC1E 6BT, U.K.

\section{Supporting Information}

ABSTRACT: Ciliated lung epithelial cells and the airway surface liquid (ASL) comprise one of the body's most important protective systems. This system is finely tuned, and perturbations to ASL rheology, ASL depth, ASL pH, the transepithelial potential, and the cilia beat frequency are all associated with disease pathology. Further, these apparently distinct properties interact with each other in a complex manner. For example, changes in ASL rheology can result from altered mucin secretion, changes in ASL $\mathrm{pH}$, or changes in ASL depth. Thus, one of the great challenges in trying to understand airway pathology is that the properties of the ASL/epithelial cell system need to be assessed nearsimultaneously and without perturbing the sample. Here, we show that nanosensor probes mounted on a scanning ion conductance microscope make this possible for the first time, without any need for labeling. We also demonstrate that ASL from senescenceretarded human bronchial epithelial cells retains its native properties. Our results demonstrate that by using a nanosensor approach, it is possible to pursue faster, more

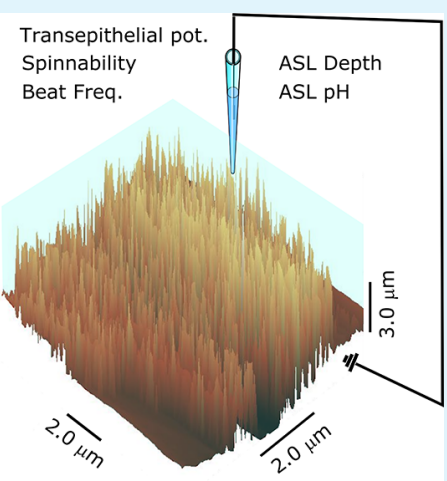
accurate, more coherent, and more informative studies of ASL and airway epithelia in health and disease.

KEYWORDS: scanning ion conductance microscopy, spinnability, nanopipette, mucociliary clearance, airway surface liquid, cystic fibrosis

\section{INTRODUCTION}

The airway surface liquid (ASL) is a very shallow $(\sim 10 \mu \mathrm{m})$, viscoelastic gel that covers the upper airways. Inhaled pathogens such as viruses and bacteria become trapped in its mucus layer and are then removed from the lungs by beating cilia. ${ }^{1,2}$ Thus, working together, the ASL and ciliated epithelia create a vital protective system for the body. Alterations in ASL depth, rheology, and acidity as well as the beating of cilia are thought to underlie serious aspects of disease pathology associated with illnesses such as cystic fibrosis (CF), asthma, cilia dyskinesias, and chronic obstructive pulmonary disorder (COPD)..$^{3-8}$

The ASL is usually thought of as being divided into two layers, an upper mucus layer and a lower periciliary liquid (PCL) within which the cilia beat. It used to be believed that the PCL was a "watery" layer to allow free motion of the cilia, whilst the mucus layer was much more viscous, important for its role in trapping pathogens. However, this idea is not consistent with the fact that $40 \mathrm{~nm}$ particles cannot penetrate the PCL layer, and recently, a revised 'gel on a brush' model has redefined thinking about these two layers. ${ }^{2}$ In this new model, the PCL layer consists of membrane-spanning mucins and mucopolysaccharides tethered to the airway surface, including the cilia themselves. ${ }^{2}$

The gold standard for studying the ASL in vitro is to use primary cultures of human bronchial epithelial (HBE) cells grown at an air-liquid interface (ALI). These cultured cells spontaneously produce ASL and retain many of their properties seen in vivo. Experiments with such cultures have shown that ASL depth, ASL rheology, ASL acidity, and cilia beat frequency $(\mathrm{CBF})$ can interact with one another in a complex manner. ${ }^{9-11}$ Therefore, to understand how disease alters the ASL, it is necessary to measure these properties nearsimultaneously. In addition, it is also useful to have a measure of the transepithelial potential, which reflects the ion driving forces that regulate ASL hydration. This potential is heavily influenced by the apical sodium conductance and the conductivity of the paracellular pathway. In CF individuals, an increased $\mathrm{Na}^{+}$conductance in the apical membrane can be an aid in diagnosis. For all of the measurements discussed above, the shallowness of the ASL presents a major technical challenge.

The technical difficulties that have to be faced when measuring the ASL mean that existing methods have important limitations and also require a range of apparatus. For example, to measure the transepithelial potential, the ASL is often flooded in order to safely place an electrode above the apical membrane without damaging it. Clearly, this precludes

Received: August 16, 2018

Accepted: January 16, 2019

Published: January 16, 2019 
subsequent depth measurements. Depth itself is usually estimated from confocal z-stacks recorded at a variety of ASL locations and thus requires ASL labelling along with timeconsuming post-imaging analysis. ${ }^{7}$ Further, perhaps the most useful rheological properties of viscoelasticity and spinnability cannot be measured in such small culture samples. To address these problems, we have developed an approach based around scanning ion conductance microscopy (SICM). SICM is a member of the scanning probe family of methods that includes atomic force microscopy and scanning electrochemical microscopy (SECM). SICM was invented by Paul Hansma, ${ }^{12}$ but its potential for biological imaging was developed first by the Korchev laboratory. ${ }^{13}$ SICM imaging relies on the current flowing between a nanopipette and a ground electrode for its feedback signal. As the probe approaches a nonconducting surface, current flow is reduced and thus SICM is capable of deriving high-resolution topographic cell images without contacting the sample. It is this feedback signal that has allowed us to develop a suite of methods for studying the ASL.

\section{RESULTS}

We have developed a fast and simple approach to the measurement of ASL and epithelial cell function that uses just a single apparatus consisting of nanoprobes mounted on a scanning ion conductance microscope (SICM). Unlike most existing methods, our approach requires no labelling and works for both transparent and opaque samples.

The arrangement of our SICM apparatus for studying ALI cultures is illustrated schematically in Figure 1a. SICM imaging a)

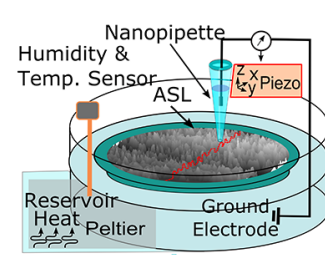

c)

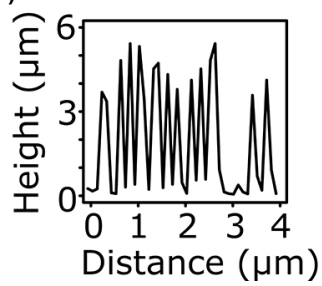

b)

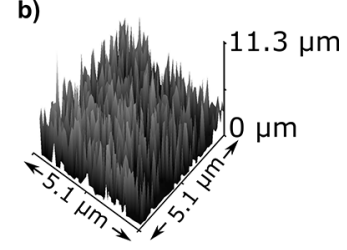

d)

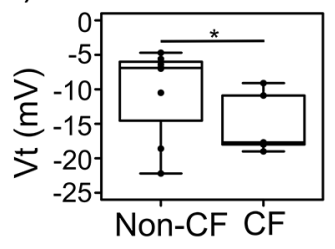

Figure 1. Experimental configuration for SICM ASL and epithelial cell measurements. (a) Schematic of the experimental configuration. An ALI culture is mounted in an environmentally controlled chamber, maintaining the cells at $99 \%$ humidity and $37^{\circ} \mathrm{C}$ via a Peltier and bath reservoir. The SICM ground electrode is situated in the basolateral medium, whereas the SICM nanopipette probe enters the ASL. (b) Image of the surface of a senescence-retarded normal human bronchial epithelia cell grown in an ALI culture. Densely packed cilia are evident. (c) Line profile taken from the cilia in (b). (d) Values of transepithelial potential $\left(V_{\mathrm{t}}\right)$ obtained from $n=8$ non-CF and $n=5 \mathrm{CF}$ cultures. Median $V_{\mathrm{t}}$ values were different $(p<0.05)$.

relies on current flowing between a ground electrode and a nanopipette probe that is filled with normal, physiological solution. Usually both of these electrodes are situated in the fluid that overlays the top of the sample, ${ }^{12,13}$ which in this case would be the ASL. However, the transepithelial resistance of an epithelial cell culture grown at an ALI is relatively low compared to that of the nanoprobe (typically $<1400 \Omega * \mathrm{~cm}^{2}$, compared to $\sim 80-120 \mathrm{M} \Omega) .{ }^{14,15}$ This means that it is possible to place the ground electrode on the basolateral side of the epithelial monolayer without adversely affecting the feedback system. Further, the salt concentrations of the ASL are believed to be close to normal physiological values for other extracellular fluids with a $\mathrm{Na}^{+}$concentration of approximately $100 \mathrm{mM}$ in both CF and non-CF individuals. ${ }^{16,17}$ Thus, pipette $I-V$ curves in the ASL are very similar to those seen in normal physiological saline (Figure S1 in the Supporting Information).

Further, when the probe first enters the ASL solution, the pipette current jumps from zero to its maximum value, almost instantaneously. This signal triggers the SICM's feedback system to halt the pipette immediately (within nanometers), and thus, an SICM probe can be lowered into the ASL automatically without having to worry about colliding with, or damaging, the cell surface. From this position (Figure 1a), it is possible to image the surface using fine nanoprobes (tips 30 $\mathrm{nm}$ radius)..$^{15,18,19}$ It is interesting to note that if the apparent height of the tallest cilia is taken as a proxy for a minimal PCL depth, then the PCL must be $>5.5 \mu \mathrm{m}$ and this value is consistent with PCL depths reported in the literature (e.g., Cho et al., PCL depth $6.72 \pm 0.4 \mu \mathrm{m}$ ).

With the configuration shown in Figure 1a, an immediate benefit of placing the ground electrode underneath the epithelial cell layer is that it makes measuring the transepithelial potential very straightforward. ${ }^{20}$ This is done by simply connecting a patch-clamp amplifier to the SICM probe and switching to the current clamp mode once the probe is immersed. We tested this approach by examining newly developed human bronchial epithelial cells, modified by lentiviral transduction with $B M I-1$ to retard senescence. ${ }^{14}$ $B M I-1$ transduction very substantially extends the proliferative potential of epithelial cells whilst retaining their capacity for mucociliary differentiation. We used senescence-retarded cells developed both from a healthy (non-CF) donor and a CF ( $\triangle$ F508-CFTR homozygous) donor. We measured the transepithelial potentials in five CF and eight non-CF ALI monolayers. Consistent with disease pathology, non-CF senescence-retarded cells were more depolarized than their CF counterparts ${ }^{21}$ (Figure 1d).

We next sought to use SICM as a tool for ASL depth measurement. We again used the fact that as the probe begins its descent toward the sample, a sudden jump in current occurs when the probe tip first touches the upper surface of the ASL fluid. This position can thus be recorded as the upper limit of the ASL. The bottom of the ASL can also be located by the probe because, when it is within $\sim 1$ radius of the cell surface, the flow of ions becomes partially occluded and the feedback current drops $^{13,19,22}$ (Figure 2a). The ASL depth is simply the difference between these two positions.

To test the reliability of this method, we measured depth changes when small volumes of saline solution $(\sim 1 \mu \mathrm{L})$ were added to $\sim 1 \mathrm{~mL}$ of the same solution in a $35 \mathrm{~mm}$ Petri dish. This is expected to produce a change of $0.1 \%$ or $\sim 1 \mu \mathrm{m}$ on top of a depth of $\sim 1000 \mu \mathrm{m}$, a major challenge for any technology. The results from this experiment are shown in Figure $2 \mathrm{~b}$. The line drawn through these data was fitted to the intercept only. The slope of the line was calculated theoretically from the depth change expected given the average weight of solution added to the sample and its cylindrical geometry. The agreement between prediction and measurement is excellent 


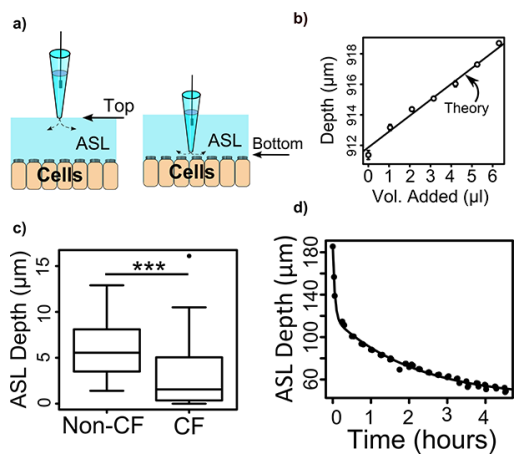

Figure 2. Measurement of ASL depth. (a) Schematic of the depth measurement protocol. Current flow begins the moment a nanopipette enters the ASL, identifying the location of the top of the fluid. When the probe approaches the cell surface, the current becomes reduced. This signal identifies the position of the bottom of the ASL. (b) Validation of depth measurement. One microliter volumes of solution were added sequentially to a starting volume of $\sim 1 \mathrm{~mL}$ (initial depth $\sim 912 \mu \mathrm{m}$ ) in a circular Petri dish. The weight of the added solution and the cylindrical geometry were used to predict the slope of the solid line (theory), which was fitted to the intercept only (overall, $R^{2}=0.978$ ). Heights (open circles) are shown with SEM (from triplicate measurement), unless smaller than the size of the symbol. (c) Box plots showing the distribution of ASL depths for two non-CF cultures (measurements at 46 locations) and two CF monolayer cultures (measurements at 40 locations). The difference in mean depths argues strongly against the null hypothesis (*** indicates $p<0.001$ ). (d) A twenty microliter of isotonic buffer was added to the center of the ASL on a non-CF monolayer. The height at that location was then followed by repeated depth measurements over a $5 \mathrm{~h}$ period. The data were fitted to a biexponential decay (weighted mean time constant of $3.4 \mathrm{~h}$ ). In five such experiments, the average of the weighted mean time constant was $4.1 \pm 1.5 \mathrm{~h}$.

$\left(R^{2}=0.978\right)$. Further, the variance in point estimates is $\sim 135 \mathrm{~nm}$, indicating a $z$-resolution well beyond that of most commonly used optical methods (which is $\sim 0.6-1 \mu \mathrm{m}$ under optimal conditions). . $^{2324}$

Having validated our method of depth measurement, we then applied it to determine the depth of the ASL produced by BMI-1-transformed HBE cells. Again, we used cells from both the healthy (non-CF) donor and the CF donor. Both sets of cells were grown at an air-liquid interface for four weeks, washed, and the ASL depth was then measured $24 \mathrm{~h}$ later. An environmental chamber was used to maintain $99 \%$ humidity and a temperature of $37{ }^{\circ} \mathrm{C}$ during measurement. The variation in depth at different surface locations was not always normally distributed (Supporting Information Figure S2). Figure $2 \mathrm{c}$ shows the median depths and quartile spread, 3.6$7.8 \mu \mathrm{m}$ for non-CF ( $n=2$ cultures, 46 measurements $)$ and 0.38-5.0 $\mu \mathrm{m}$ for CF cultures $(n=2$ cultures, 40 measurements). Calculating the mean depths for comparison to literature values gives depths of $5.8 \pm 0.4$ and $3.0 \pm 0.5 \mu \mathrm{m}$ for the non-CF and CF cultures, respectively. Both of these values of ASL mean depth and the observation of a shallower ASL in CF cultures $(p<0.001)$ agree very well with data obtained from primary cultures $(\sim 7 \mu \mathrm{m}$ for non-CF and $\sim 3 \mu \mathrm{m}$ for CF cultures measured by confocal microscopy). ${ }^{25,26}$

We have also examined the ability of our system to follow dynamic ASL depth changes by adding $20 \mu \mathrm{L}$ of saline to the center of a non-CF ALI culture and following absorption. In this experiment, cells were maintained in their environmental chamber and the change in ASL height was followed at a fixed position near the center of the culture for several hours. Typically, data obtained in this way were well described by a biexponential absorption curve (Figure 2d). The mean weighted time constant of these absorption curves was $4.1 \pm$ $1.5 \mathrm{~h}$ (absorption measured in $n=5$ cultures, Supporting Information Figure S3). This meant that ASL height returned to a value close to normal after $\sim 24 \mathrm{~h}$, consistent with reports using other methods applied to cultures that were not BMI-1treated. ${ }^{25}$ Thus, in both steady-state ASL depth and ASL absorption rates, the behavior of BMI-1-transformed HBE cells appears similar to that reported for untransformed cells using alternative methods.

We next sort to measure CBF. Because contact-free SICM imaging relies on partial obstruction of the nanoprobe tip, we reasoned that when the probe tip reaches beating cilia, it should be possible to see periodic oscillations in the feedback signal (Figure 3a). We tested this approach by again using air-

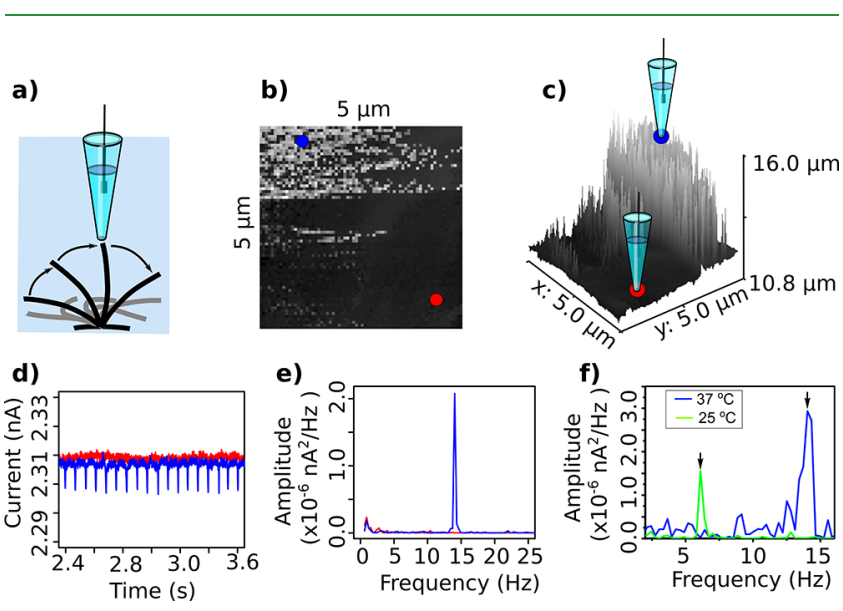

Figure 3. CBF measured by SICM. (a) Schematic of cilia interaction with the nanoprobe. The probe is only sensitive when the outstretched cilia are very close to its tip (within approximately one times the inner probe radius). (b) Scan of the epithelial cell surface viewed from above. Height is indicated by gray scale (lighter is higher). The locations of the nanopipette when recording from ciliated and unciliated regions are indicated by the blue and red dots, respectively. (c) 3D image from the scan shown in (b) with locations of recording sites. (d) Current traces when the probe is located over ciliated (blue trace) and unciliated (red trace) regions of $\mathrm{b} / \mathrm{c}$. Only ciliated regions show periodic oscillations. Similar observations were made in three separate cultures. (e) Power spectrum of the current traces in (d). The recording from a ciliated region has a clear peak at $\sim 14 \mathrm{~Hz}$, whereas there is no peak after recording from an unciliated region. (f) Power spectrum of current recordings taken from a single ciliated region at $37{ }^{\circ} \mathrm{C}$ (blue) and $25{ }^{\circ} \mathrm{C}$ (green). Peak frequency drops from $\sim 14$ to $\sim 6.1 \mathrm{~Hz}$ at the lower temperature. Similar observations were made in three cultures.

liquid interface cultures of the senescence-retarded HBE cells, this time using non-CF cells. As a first step, we used the SICM to obtain a high-resolution image of the sample surface so that regions of both ciliated and unciliated cells could be clearly identified. An example of this type of scan is shown in Figure $3 b, c$. Using the scan, we positioned our probe over both ciliated and unciliated regions of the culture surface. In each $\mathrm{CBF}$ recording, we first positioned the probe high above the cilia before subsequently lowering it. When the probe was high above the beating cilia, no signal was seen. However, when the probe was lowered on top of a ciliated region, a clear periodic signal became evident (Figure $3 \mathrm{~d}$ ). This beating signal 
vanished when the probe was withdrawn, and no such signal was seen when the probe was either high above, or close to, an unciliated cell area (Figure $3 \mathrm{~d}$ ). We conclude that current oscillations are caused by beating cilia. Taking a power spectrum of the probe, current gives the beat frequency (Figure $3 \mathrm{e}$ ). Using this method, we found beat frequencies in the range $\sim 8-14 \mathrm{~Hz}$ ( $n=3$ cultures) at $37{ }^{\circ} \mathrm{C}$ for senescenceretarded cells. These values agree well with measurements using video microscopy-based techniques both in the same cells $^{14}$ and in standard primary cultures. ${ }^{27,28}$ Furthermore, lower temperatures are well known to reduce beat frequency ${ }^{28}$ and, as shown in Figure 3f, lower temperatures reduced CBF values in our BMI-1-transformed cells from 8-14 to $3-8 \mathrm{~Hz}$ ( $n=3$ cultures) as expected.

We next set out to measure spinnability (the ability to pull a thread of solution). ASL spinnability is one of the most useful rheological measures because it is negatively correlated with both predicted mucociliary clearance and cough clearance. ${ }^{29}$ To measure spinnability, the probe tip was immersed just under the surface of the ASL and then withdrawn. The point at which the ASL thread snaps during withdrawal can be precisely determined by the SICM via the loss of current flow (Figure 4a).

a)
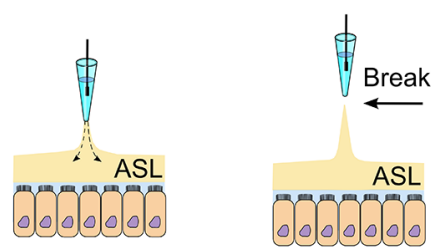

b)

c)
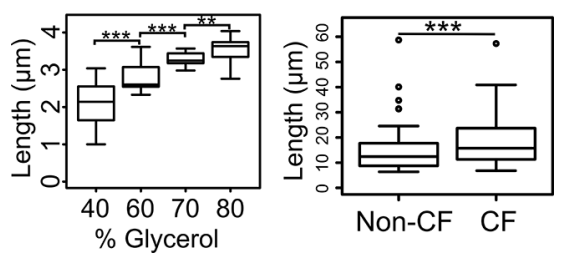

Figure 4. Measurement of ASL spinnability. (a) Schematic of SICMbased spinnability measurement. The nanoprobe is inserted into the ASL and then withdrawn at a constant speed. A thread of solution forms before it eventually breaks, cutting the electrical circuit between the nanoprobe and the bath electrode. The length of the thread provides the measure of spinnability. (b) Spinnability control measurements using glycerol solutions. The mean length of the thread increases with the percentage of glycerol in solution $(* * * p<$ $0.001, * * p=0.01), n=15$ measurements per solution. (c) Combined ASL spinnability data from $6 \mathrm{CF}$ and 6 non-CF monolayer cultures using BMI-1-transformed HBE cells. CF cultures show an increased mean ASL spinnability $(p<0.001)$.

To confirm the validity of our approach, we examined a range of glycerol solutions. The results in Figure $4 \mathrm{~b}$ clearly show that as the percentage of glycerol is increased (and hence the viscosity increases), the mean spinnability increased. We applied this technique to senescence-retarded cells from both $\mathrm{CF}$ and non-CF lineages. A total of 12 cultures were used (6 $\mathrm{CF}$ and 6 non-CF), sampling at 12-21 locations on each culture. Spinnability was variable within and across cultures but consistent with the difficulty in mucociliary and cough clearance that is seen in CF disease; the ASL in CF cultures had a higher mean spinnability than the non-CF cultures $(p<$ 0.001 ; Figure 4c).

Finally, we examined the possibility of measuring ASL $\mathrm{pH}$. To measure $\mathrm{pH}$, we manufactured a $\mathrm{pH}$-sensitive electrode by filling the tip of a single-barrel SICM probe with a protonselective cocktail (Selectophore I cocktail) (Figure 5a). This

a)

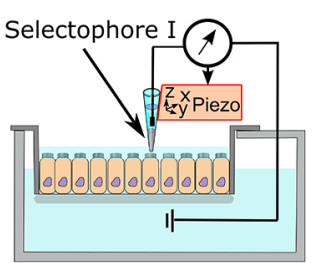

b)
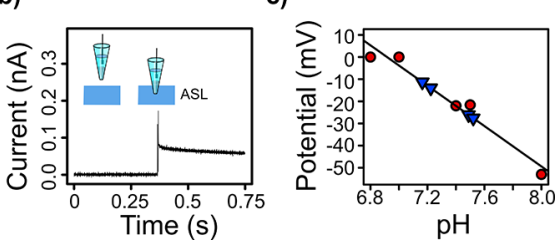

Figure 5. Measurement of ASL pH. (a) Schematic of ASL pH measurement. A pH electrode is created by filling the SICM probe tip with a $\mathrm{H}^{+}$-selective medium (Selectophore I, cocktail) illustrated in gray. The SICM probe is connected to a patch-clamp amplifier which is set to current clamp for measurements. (The transepithelial potential of the culture must be recorded with a standard probe and subtracted from the $\mathrm{pH}$ electrode value.) (b) ASL immersion of a Selectophore-filled probe produces a small but obvious current transient, sufficient to trigger the SICM control software to stop the probe's descent. (c) Estimates of ASL $\mathrm{pH}$ for $n=4$ different non-CF cultures (blue triangles) estimated from the line of best fit (solid line) to probe measurements made against standard solutions (red circles).

hugely reduces the current that flows when the probe is immersed, but there remains a transient current upon entering the ASL which is enough to provide the feedback necessary to halt the pipette as soon as it contacts the solution (Figure $5 \mathrm{~b}$ ). A patch-clamp amplifier connected to the probe was used to record the potential for comparison with potentials from $\mathrm{pH}$ standards (after subtracting the transepithelial potential measured as above). Applying this method to our senescence-retarded HBE cells, we measured a mean $\mathrm{pH}$ value of $7.35 \pm 0.09$ from 4 non-CF cultures (Figure $5 \mathrm{c}$ ), close to the $\mathrm{pH}$ value in ambient $\mathrm{CO}_{2}$ reported by others. ${ }^{30}$

\section{DISCUSSION}

It has long been established that it is defects in the gene encoding the cystic fibrosis transmembrane regulator (CFTR) protein that results in CF. However, the link between a lack of activity in the CFTR anion channel and the resulting bacterial lung infections remains controversial. Recently, two main ideas have been most prominent in the literature, and both concern changes to the ASL. The first suggestion is that dehydration of the ASL, likely driven by an increased $\mathrm{Na}^{+}$permeability on the apical membrane of the epithelia, prevents effective mucociliary transport. ${ }^{1}$ The second proposal is that changes in ASL pH are responsible. ${ }^{30}$ However, these ideas are not without criticism $^{31,32}$ and much remains to be understood about the ASL and its role in CF pathology. To aid in this work, we have developed a nanosensor/SICM approach that allows measurement of transepithelial potentials, ASL depth, ASL spinnability, 
a)

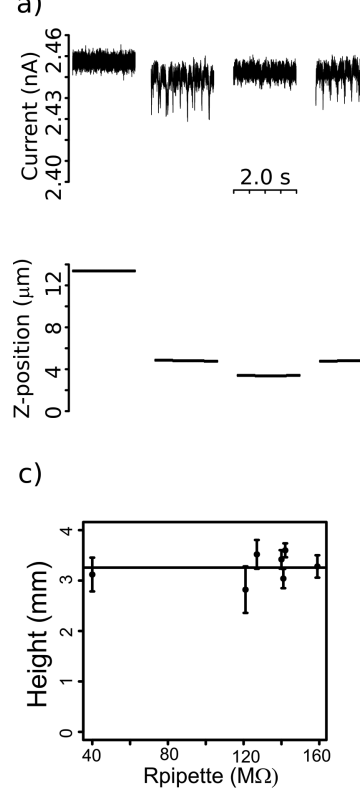

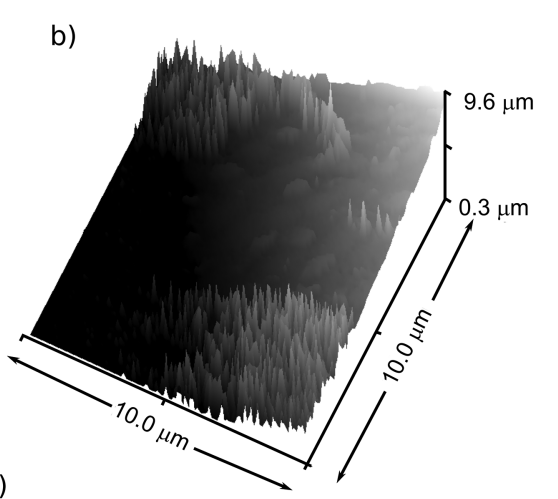

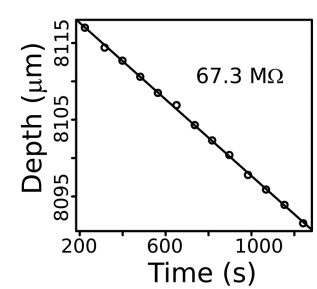

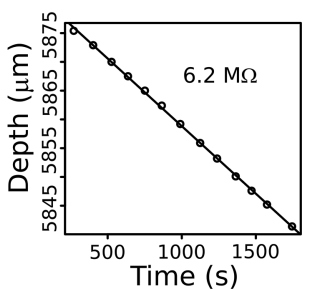

Figure 6. Robustness of pipette measurements. (a) Variation in signal vs pipette position for the beating action of cilia. When the pipette is high above the cilia (trace far left and trace far right), no beating is seen. When it is lowered to the sample surface, beating is clearly evident (traces second from left and second from right). Lowering the pipette further diminishes, but does not completely eliminate, a signal (middle trace). (b) Scan of the epithelial cell surface just below the recordings shown in (a). For recording beat frequency, the pipette was positioned over cilia at the bottom right corner. (c) Spinnability recorded with pipettes of varying size $(\sim 40-160 \mathrm{M} \Omega)$ using a standard solution of $0.09 \mathrm{mg} / \mathrm{mL}$ collagen. The mean spinnability averaged from all pipettes is shown by the horizontal black line. There is no evidence of a trend with pipette size over this range. (d) Height changes seen as the solution evaporates from a Petri dish. Both fine (67.3 M $\Omega$, left panel) and large (6.2 M $\Omega$, right panel) pipettes perform well. Solid lines are fitted to the data and predict evaporation rates of $24.3 \pm 0.1$ and $23.5 \pm 0.1 \mathrm{~nm} / \mathrm{s}$ (assuming a cylindrical solution geometry). Actual rates of loss assessed by changes in the weight of solution and assuming the same geometry were 23.5 and 23.1 nm/s, within $\sim 3 \%$ of the measured values.

ASL pH, CBF, and even a lower limit for PCL depth. Our approach can accelerate ASL measurement and, through spinnability, allows measurements that were not previously possible with small samples. Indeed, the development of such small-scale spinnability measurements may be useful to those interested in the rheology of other materials. In addition, the nanoprobe method we use for depth measurement offers higher resolution than most standard techniques and the ability to make multiple types of measurements from a single culture/ sample dramatically reduces the need for human tissue and thus also reduces costs. Indeed, costs were part of the motivation for deriving the BMI-1-transformed cells. In the current work, we have found that these cells preserve many of the properties of their source phenotype and may thus form an important tool in the arsenal of approaches for tackling airway disease. Together, BMI-1 cells and SICM methods offer a number of advantages when studying the ASL physiology and/ or the effects of medicines targeting the ASL. Further, by using twin barrel SICM probes, for example, for scanning and $\mathrm{pH}$ measurement, ${ }^{33,34}$ and implementing software automation and faster scanning, ${ }^{35}$ there are obvious ways to increase the speed and versatility of these methods even further.

Our work demonstrates that the imaging capability of SICM can be used to detect large, motile cilia and this raises the possibility of using SICM imaging for comparative studies of airway cilia size, which have shown value in understanding lung pathology. ${ }^{36}$ From our SICM images, cilia appear to vary in height (typically $\sim 3-5 \mu \mathrm{m}$, Figure 1c) and similar variability has often been reported in the literature. For example, Li et al. ${ }^{37}$ used tubulin staining to measure cilia length from human nasal epithelia and estimated lengths of $\sim 3.50-4.27 \mu \mathrm{m}$. Shah et al. ${ }^{38}$ also used light microscopy and estimated an average length of $4.1 \mu \mathrm{m}$ in ALI cultures of mouse airway epithelia. However, several other measures put the value closer to $6 \mu \mathrm{m}$ or slightly larger. ${ }^{36}$ In the intact airways, Serafini and Michaelson $^{39}$ found that cilia length varies with the location of the epithelia from $\sim 6.0 \mu \mathrm{m}$ in the trachea to $3.6 \mu \mathrm{m}$ in the lower airways, which may account for some of these differences. In contrast to the varying lengths that have been reported, most studies agree that the width of an individual cilium is $\sim 200-300 \mathrm{~nm} .{ }^{40}$ Taken overall, there is a reasonable level of agreement between our SICM images and values of cilia size reported in the literature. Thus, it may perhaps be possible to do comparative studies in the future. However, it is important to guard against over-interpreting the images. Interactions between SICM imaging and cilia movement (Supporting Information Figure S4) inevitably complicate measurement and will lead to some underestimation of cilial length. Further, both theoretical and practical studies have shown that considerable care is needed to avoid SICM imaging distorting the widths of individual features when encountering vertical edges such as those that may be created by the cilial layer. ${ }^{19}$ Thus, comparative cilia size studies may be possible but care will be needed, even if fixed or nonmotile types of cilia are used, as others have pointed out. ${ }^{41,42}$

Although we have demonstrated the capability of our techniques, it is of interest to examine some of the limitations too, in order to clarify their utility further. Returning first to $\mathrm{CBF}$, we looked to see if the signal was affected by flooding/ washing the sample to remove any gradients in fluid/ionic composition or viscosity that may exist between the PCL and mucus layers and could perhaps affect the SICM signal. We 
found that a flooded sample from the rat trachea still produced a strong beat signal (Supporting Information Figure S5). We then examined how varying the pipette position affected the beating signal. We find there is a 'sweet spot' for detecting beating and if the probe continues to descend beyond this region, the signal becomes weaker (Figure 6a). We interpret this as the probe reaching a position below the beating tips of the cilia. These observations suggest that the beating signal we see is the result of direct interaction between the probe and the cilia.

We have also examined the impact of varying the pipette size on our system of measurements. Whilst large pipettes are quite practical for measuring $\mathrm{pH}$, it is less clear that they will work for some of the other approaches we have developed. For measuring cilia beating, testing large pipettes is complicated by the fact that large pipettes do not have high enough resolution to clearly visualize cilia below the probe and thus to ensure that the correct region is being recorded from. However, we were unable to detect cilia beating when attempting to place a large patch pipette $(\sim 5 \mathrm{M} \Omega$ resistance) over regions where fine pipettes produced a clear signal (data not shown). We therefore believe that large pipettes may not be suitable for this measurement.

For examining the impact of pipette size on spinnability, we used a saline containing $0.09 \mathrm{mg} / \mathrm{mL}$ collagen. This creates a solution dominated by elasticity (in line with the properties of the ASL). Using glass with a constant ID/OD ratio, we find that our spinnability measure is robust over the range of pipette sizes tested (40-160 M $\Omega$, Figure 6b).

We next tested the ability to follow depth changes using both small and large pipettes. To examine this issue, we followed the reduction in solution depth brought about via the evaporation of saline from a partly covered Petri dish. This gave depth changes of about $\sim 24 \mathrm{~nm} / \mathrm{s}$. We find that even patch-size pipettes used with SICM technology can follow evaporation with great accuracy in control situations (Figure $3 b, c)$. Such pipettes could therefore be used to measure fluid absorption in ASL cultures. However, using large pipettes has several important limitations. First, the $x-y$ resolution in depth measurements is poor because large pipettes cannot get between cilia, meaning that they are likely to underestimate the depth. (For this same reason, they are unlikely to provide a good way to estimate a minimum PCL depth.) Second, SICM theory dictates that with typical set points, the pipette will stop at about one radius above the surface membrane. For large pipettes, this value will start to have a significant impact on the absolute depth. The sloping cell surfaces will also be a problem, making large pipettes less accurate. Such a large pipette approach would be similar to the electrode method pioneered by Rahmoune and Shephard, ${ }^{43}$ but by implementing SICM control and using smaller pipettes, faster and more accurate measurement is possible. Further, SICM feedback control in combination with nanopipettes avoids the probe pushing directly down on the cell membrane surface, which can cause cell retraction and thus create complications in the measurement process. Thus, from the experiments described above, we believe that with the exception of $\mathrm{pH}$ and to some extent spinnability, nanopipettes offer advantages for studying the ASL.

Many methods have been developed for measuring ASL depth and/or ASL volume, with volume and depth tending to show qualitatively similar responses to drugs or other types of intervention. ${ }^{44}$ The most common of these is the use of confocal imaging, ${ }^{7}$ but ASL depth or volume can also be measured by micro-optical coherence tomography (discussed below), surface laser reflectance microscopy, ${ }^{45}$ deuterium oxide dilution, ${ }^{46}$ and light refraction microscopy. ${ }^{44}$ All of these methods have specific advantages and disadvantages. The deuterium method obviously requires handling radioactivity, and at least in our hands, the reflectance microscopy signal is somewhat complicated by the ciliated surface of the epithelia. Of course, our SICM method measures ASL fluid depths whether it is between the surface of the ASL to the tips of the cilia or from the surface of the ASL to the base of the cilia. If it is only the latter that is desired, a 3D scan can be used to clarify the situation. However, estimating the 'true' ASL depth (from all points on the cell membrane to the surface of the fluid) may be desirable when there are many cilia and the aim is to introduce defined drug concentrations, or known concentrations of signaling molecules. In confocal imaging, which is by far the most popular approach, the cilia are often not stained/visible and it may be a challenge to know whether the ASL is being measured in a ciliated or unciliated region. Further, with any imaging approach, it is important to avoid the bias that can occur when a user selects the regions to be analyzed by viewing the images first. Because SICM imaging is done "blind", it perhaps offers a small advantage over some of the methods described above.

Much work has also been devoted to developing optical techniques for measuring the other ASL properties, for example, $\mathrm{Na}^{+}$concentrations or $\mathrm{pH} .{ }^{47}$ However, these require ASL dye loading, and the difficulty measuring a full range of ASL properties on a single culture remains significant. Interestingly, motivated to improve ASL measurement, Tearney and Rowe developed and applied $\mu \mathrm{OCT}^{24,48}$ to this problem. Their important work has created another way to perform several ASL/epithelial cell measurements without labelling, including ASL depth, PCL depth, CBF, and mucocilliary transport rates. However, $\mu \mathrm{OCT}$ has lower resolution $(\sim 1 \mu \mathrm{m}$ in $x, y$, and $z)$ than $\operatorname{SICM}^{48}$ and cannot measure ASL $\mathrm{pH}$, transepithelial potential, or ASL spinnability. In addition, our $\mathrm{pH}$ recordings represent just one possible example of using an ion-selective electrode. Other types of ionselective electrode (e.g., $\mathrm{K}^{+}$-selective electrodes ${ }^{43}$ ) could equally well be used if desired. Further, our SICM methods can also be extended by combining them with optical methods, ${ }^{49}$ SECM techniques, ${ }^{50-52}$ and novel SICM configurations for studying the paracellular pathway. ${ }^{20}$ We believe that this versatility will make SICM an essential tool for studying ASL/epithelial cell properties.

\section{CONCLUSIONS}

We have developed a simple, single platform nanosensor approach to the measurement of ASL and epithelial cell function that is faster, more convenient, and more informative than many existing approaches. Further, it can be adapted for use with both opaque and transparent samples. Using this nanosensor technology, we have shown that HBE cells with delayed senescence retain healthy (non-CF) and diseased (CF) ASL/epithelial cell phenotypes. Together, these technologies can help to transform the availability of human epithelial cells and can greatly accelerate the study of major diseases where the ASL and/or mucociliary transport are affected, such as CF, COPD, and asthma. 


\section{METHODS}

Air Liquid Interface Cultures. Non-CF and CF ( $\triangle$ F508 homozygous) $\mathrm{HBE}$ cells, transformed with BMI-1 to delay senescence, were maintained in BEGM medium (Lonza) supplemented with $100 \mathrm{u} / \mathrm{mL}$ penicillin and $100 \mu \mathrm{g} / \mathrm{mL}$ streptomycin and 1 $\mu \mathrm{g} / \mathrm{mL}$ fungizone (Life Technologies). Cells were used at passages 10-17 and were seeded onto collagen-coated (bovine collagen solution, $3 \mathrm{mg} / \mathrm{mL}$, Purecol) $12 \mathrm{~mm}$ snap-well polyester membranes (Corning 3407, $0.4 \mu \mathrm{m}$ pore). A minimum of 1 million cells was seeded per well in $250 \mu \mathrm{L}$ BEGM medium. The basolateral side of the membrane was bathed in $2 \mathrm{~mL}$ of BEGM for the initial $48 \mathrm{~h}$. At that point, air-liquid interface (ALI) was established by aspirating the apical medium, leaving a confluent monolayer of cells exposed to air. The basolateral medium was replaced with an ALI medium composed of a 1:1 ratio of BEBM (Lonza) and DMEM (Life Technologies), supplemented with a Bullet Kit (Lonza), $100 \mathrm{u} / \mathrm{mL}$ penicillin and 100 $\mu \mathrm{g} / \mathrm{mL}$ streptomycin (Life Technologies), $1 \mu \mathrm{g} / \mathrm{mL}$ fungizone (Life Technologies), and $100 \mathrm{nM}$ retinoic acid (Sigma). Cells were fed by replacing the basolateral medium three times a week. Cells were cultured for 3-6 weeks to allow differentiation and formation of cilia.

Trachea. When testing the ability of large pipettes to detect cilia beating, some additional experiments were performed using rat trachea. For these experiments, Male Sprague Dawley Rats (250-350 g) were killed by $\mathrm{CO}_{2}$ asphyxiation in accordance with local laws (UK Animals (Scientific Procedures) Act 1986). The trachea was rapidly removed and transferred to Ringer solution at room temperature. A longitudinal cut was made in the trachea to allow it to be opened, and it was pinned, luminal surface upward, to the bottom of a $35 \mathrm{~mm}$ Petri dish coated with SYLGARD 184 Resin (Corning), and the dish was flooded with standard saline $(116 \mathrm{mM} \mathrm{NaCl}, 10 \mathrm{mM} \mathrm{NaHCO} 3,5.1$ $\mathrm{mM} \mathrm{KCl}, 1.2 \mathrm{mM} \mathrm{MgCl}, 1.2 \mathrm{mM} \mathrm{CaCl}, 20 \mathrm{mM}$ HEPES, and 10 $\mathrm{mM}$ glucose).

Probe Fabrication. Standard SICM nanopipettes were pulled using a P2000 laser puller (Sutter Instruments, USA) and fabricated from a filamented borosilicate glass (Harvard Apparatus, O.D. $1 \mathrm{~mm}$, I.D. $0.58 \mathrm{~mm}$ ). These probes had resistances of 60 to $150 \mathrm{M} \Omega$ when filled with phosphate-buffered saline (PBS) composed of $125 \mathrm{mM}$ $\mathrm{NaCl}, 4.2 \mathrm{mM} \mathrm{KCl}, 1.2 \mathrm{mM} \mathrm{MgCl}_{2}, 1.2 \mathrm{mM} \mathrm{CaCl}_{2}, 0.8 \mathrm{mM} \mathrm{K}_{2} \mathrm{HPO}_{4}$ $\mathrm{pH}$ 7.4. The tip radius of these pipettes was $\sim 30-50 \mathrm{~nm}$. ${ }^{15}$ For $\mathrm{pH}$ probes and large pipette experiments, low resistance (1-5 M $\Omega$ ) borosilicate pipettes were pulled from glass $(1.5 \mathrm{~mm}$ O.D, $1.17 \mathrm{~mm}$ I.D. GC150TF; Harvard Apparatus) on a two-stage vertical puller (PC-10, Narishige). After pulling, $\mathrm{pH}$ probes were baked at $200{ }^{\circ} \mathrm{C}$ for a minimum of $20 \mathrm{~min}$ inside a glass Petri dish within a fume hood. Silanising agent TMSDMA ( $40 \mu \mathrm{L}$; Sigma) was then added to a small glass container (at room temperature), which was then placed near the probe tips inside the glass Petri dish. Probes were then baked for an additional $15 \mathrm{~min}$ at $200{ }^{\circ} \mathrm{C}$. Finally, the lid was removed, and the pipettes were baked for another $5 \mathrm{~min}$ to allow the TMSDMA to evaporate. Once cooled, $\sim 2 \mu \mathrm{L}$ of $\mathrm{H}^{+}$-selective Selectophore I cocktail (Sigma) was added to the probe before back filling with PBS solution described above.

Imaging and Current Recording. All SICM imaging was carried out in a "hopping" mode using a scanning ion conductance microscope (ICnano S2 controller), running ICnano2000 software (OpenIOLabs) attached to an Axopatch $200 \mathrm{~B}$ patch clamp amplifier (Molecular Devices). The scan set point was $0.4 \%$, and the surface was sampled at $100 \mathrm{~nm}$ intervals. Cells were maintained in an environmental chamber at $99 \%$ humidity and $37{ }^{\circ} \mathrm{C}$. Signals were digitized via a Digidata 1440A (Molecular Devices) and written to the computer using pClamp (current traces, and pipette $z$-position) or ICnano2000 software (images). For plotting, image files were exported to, and rendered in, Gwyddion (http://gwyddion.net/)

Cilia Beat Frequency. CBF was measured with the SICM feedback switched off and either the 'approach curve' mode was used or the position of the probe was controlled manually. A power spectrum of the current was generated using Clampfit (Molecular Devices) and exported to the $R$ environment (https://www.r-project. $\operatorname{org} /)$.
Transepithelial Potentials. Transepithelial potentials were recorded using an Axopatch 200B amplifier connected to the SICM probe and set to current clamp mode $(I=0)$.

Depth Measurement Calibration. Saline $(1 \mathrm{~mL} ; 150 \mathrm{mM} \mathrm{NaCl}$ with $10 \% \mathrm{w} / \mathrm{w}$ Triton X-100) was added to a $35 \mathrm{~mm}$ Petri dish and overlaid with $2 \mathrm{~mL}$ of mineral oil (Sigma) to prevent evaporation. Height changes were then measured following the addition of $1 \mu \mathrm{L}$ volumes of solution. Alternatively, there was no oil overlay, and depth changes were measured over time with a small circular hole cut in the lid of the $35 \mathrm{~mm}$ Petri dish (33.82 $\mathrm{mm}$ internal diameter) to allow evaporation and probe access. In this case, the solution was a standard saline $(116 \mathrm{mM} \mathrm{NaCl}, 10 \mathrm{mM} \mathrm{NaHCO}, 5.1 \mathrm{mM} \mathrm{KCl}, 1.2 \mathrm{mM}$ $\mathrm{MgCl}_{2}, 1.2 \mathrm{mM} \mathrm{CaCl}_{2}, 20 \mathrm{mM}$ HEPES, and $10 \mathrm{mM}$ glucose) with $10 \% \mathrm{w} / \mathrm{w}$ Triton $\mathrm{X}-100$. All experiments were carried out at room temperature $\left(\sim 22-25{ }^{\circ} \mathrm{C}\right)$. Measured depth changes were compared to those expected based on the weight of solution lost and the dish geometry.

ASL pH Measurements. The $\mathrm{pH}$ standards at $6.8,7,7.4,7.5$, and 8 were made from a standard saline (as above). Solutions were titrated using a Corning $120 \mathrm{pH}$ meter calibrated using $\mathrm{pH} 7$ and $\mathrm{pH}$ $8 \mathrm{pH}$ standards (Sigma). Electrode responses to these solutions were recorded at room temperature $\left(\sim 23-25^{\circ} \mathrm{C}\right)$. ASL $\mathrm{pH}$ measurements were made at $37{ }^{\circ} \mathrm{C}$ and $99 \%$ humidity. A small correction was then applied to account for the temperature sensitivity of the electrode buffer.

Spinnability Calibration Measurements. The pipette was automatically lowered to $3 \mu \mathrm{m}$ below the fluid surface by the piezo motor. The pipette was then withdrawn under coarse motor control, until the filament broke. The break position was recorded using screen capture software, and the break point was taken to be the position when the system no longer considered the pipette to be immersed ( $<1 \mathrm{nA}$ of current flowing). Sampling occurred at 12 to 21 locations per monolayer. All experiments on epithelial cultures were carried out within the environmental chamber $\left(\right.$ at $37^{\circ} \mathrm{C}$ ). Aqueous glycerol (Sigma, 99\%) solutions were made up in a standard PBS solution detailed above. Experiments on model fluids were done at room temperature, whereby $1-2 \mathrm{~mL}$ of fluid was added to a $35 \mathrm{~mm}$ Petri dish (VWR) immediately prior to the start of measurements. For examining the variation in spinnability versus pipette size, a standard solution containing $150 \mathrm{mM} \mathrm{NaCl}$ and $0.09 \mathrm{mg} / \mathrm{mL}$ bovine collagen (Purecol; Advanced BioMatrix) was used. In this case, probes were manufactured using the P2000 puller as described above.

Statistics. For transepithelial potentials, ASL depths and spinnability lengths (means or medians) were compared via oneway permutation tests, applied using the "Coin" package for $\mathrm{R}$ (https://www.r-project.org/) or by using equivalent in-house software written in R. Values of $R^{2}$ were calculated as one, minus the sum of the squared difference between the observed height and predicted height divided by the sum of the squared difference between the observed heights and the mean observed height.

\section{ASSOCIATED CONTENT}

\section{Supporting Information}

The Supporting Information is available free of charge on the ACS Publications website at DOI: 10.1021/acsami.8b14122.

Comparative $\mathrm{I}-\mathrm{V}$ curves for the same nanopipette in normal physiological saline and then ASL fluid; distribution of ASL depths sampled from the surface of BMI-1--transformed human epithelial cell cultures; absorption data from five different non-CF air-liquid interface cultures; interactions between cilia beating and SICM imaging; and recording of beating cilia from a flooded trachea tissue sample (PDF)

\section{AUTHOR INFORMATION}

\section{Corresponding Author}

*E-mail: g,moss@ucl.ac.uk. 


\section{ORCID $\odot$}

\section{Guy W. J. Moss: 0000-0002-0192-6353}

\section{Author Contributions}

R.I. designed the environmental chamber, wrote the control software, and performed most of the experiments and data analysis. D.C.H.B. helped to perform the $\mathrm{pH}$ measurements and also designed and built the control electronics for the environmental chamber as well as undertaking experiments on variable pipette size with S.R. M.M.M. and S.L.H provided the BMI-1-transformed cells, assisted in developing the culture system, and helped with the early experiments. G.W.J.M. and R.I. designed the experiments. G.W.J.M. wrote the manuscript and all authors critically reviewed the manuscript before submission.

\section{Notes}

The authors declare the following competing financial interest(s): Dr. Ivanova's studentship was part funded by OpenIOLabs (now part of Cronin Group Plc) who sell scanning ion conductance microscopes).

\section{ACKNOWLEDGMENTS}

This work was supported by a BBSRC CASE studentship to R.I. co-funded by OpenIOLabs (previously Ionscope and now part of the Cronin Group) who sell scanning ion conductance microscopes and by a grant from the CF Foundation. We thank Dr. Paola Vergani, Dr. Emily Langron, and Stella Prins for helpful discussions and comments on the manuscript and Dr. Giovanna de Fillipi for excellent technical support.

\section{REFERENCES}

(1) Boucher, R. C. Airway Surface Dehydration in Cystic Fibrosis: Pathogenesis and Therapy. Annu. Rev. Med. 2007, 58, 157-170.

(2) Button, B.; Cai, L.-H.; Ehre, C.; Kesimer, M.; Hill, D. B.; Sheehan, J. K.; Boucher, R. C.; Rubinstein, M. A Periciliary Brush Promotes the Lung Health by Separating the Mucus Layer from Airway Epithelia. Science 2012, 337, 937-941.

(3) Bombieri, C.; Benetazzo, M.; Saccomani, A.; Belpinati, F.; Gilè, L. S.; Luisetti, M.; Pignatti, P. F. Complete Mutational Screening of the CFTR Gene in 120 Patients with Pulmonary Disease. Hum. Cytogenet. 1998, 103, 718-722.

(4) Fischer, H.; Widdicombe, J. H. Mechanisms of Acid and Base Secretion by the Airway Epithelium. J. Membr. Biol. 2006, 211, 139150.

(5) Innes, A. L.; Carrington, S. D.; Thornton, D. J.; Kirkham, S.; Rousseau, K.; Dougherty, R. H.; Raymond, W. W.; Caughey, G. H.; Muller, S. J.; Fahy, J. V. Ex Vivo Sputum Analysis Reveals Impairment of Protease-Dependent Mucus Degradation by Plasma Proteins in Acute Asthma. Am. J. Respir. Crit. Care Med. 2009, 180, 203-210.

(6) Bush, A.; Payne, D.; Pike, S.; Jenkins, G.; Henke, M. O.; Rubin, B. K. Mucus Properties In Children With Primary Ciliary Dyskinesia. Chest 2006, 129, 118-123.

(7) Choi, H.-C.; Kim, C. S. K.; Tarran, R. Automated Acquisition and Analysis of Airway Surface Liquid Height by Confocal Microscopy. Am. J. Physiol. Lung Cell Mol. Physiol. 2015, 309, L109-L118.

(8) Garcia-Caballero, A.; Rasmussen, J. E.; Gaillard, E.; Watson, M. J.; Olsen, J. C.; Donaldson, S. H.; Stutts, M. J.; Tarran, R. SPLUNC1 Regulates Airway Surface Liquid Volume by Protecting ENaC from Proteolytic Cleavage. Proc. Natl. Acad. Sci. U.S.A. 2009, 106, 1141211417.

(9) Tang, X. X.; Ostedgaard, L. S.; Hoegger, M. J.; Moninger, T. O.; Karp, P. H.; McMenimen, J. D.; Choudhury, B.; Varki, A.; Stoltz, D. A.; Welsh, M. J. Acidic PH Increases Airway Surface Liquid Viscosity in Cystic Fibrosis. J. Clin. Invest. 2016, 126, 879-891.
(10) Button, B.; Okada, S. F.; Frederick, C. B.; Thelin, W. R.; Boucher, R. C. Mechanosensitive ATP Release Maintains Proper Mucus Hydration of Airways. Sci. Signal. 2013, 6, ra46.

(11) Garland, A. L.; Walton, W. G.; Coakley, R. D.; Tan, C. D.; Gilmore, R. C.; Hobbs, C. A.; Tripathy, A.; Clunes, L. A.; Bencharit, S.; Stutts, M. J.; et al. Molecular Basis for PH-Dependent Mucosal Dehydration in Cystic Fibrosis Airways. Proc. Natl. Acad. Sci. U.S.A. 2013, 110, 15973-15978.

(12) Hansma, P.; Drake, B.; Marti, O.; Gould, S.; Prater, C. The Scanning Ion-Conductance Microscope. Science 1989, 243, 641-643.

(13) Korchev, Y. E.; Bashford, C. L.; Milovanovic, M.; Vodyanoy, I.; Lab, M. J. Scanning Ion Conductance Microscopy of Living Cells. Biophys. J. 1997, 73, 653-658.

(14) Munye, M. M.; Shoemark, A.; Hirst, R. A.; Delhove, J. M.; Sharp, T. V.; McKay, T. R.; O’Callaghan, C.; Baines, D. L.; Howe, S. J.; Hart, S. L. BMI-1 Extends Proliferative Potential of Human Bronchial Epithelial Cells Whilst Retaining Their Mucociliary Differentiation Capacity. Am. J. Physiol. Lung Cell Mol. Physiol. 2017, 312, L258-L267.

(15) Caldwell, M.; Del Linz, S. J. L.; Smart, T. G.; Moss, G. W. J. Method for Estimating the Tip Geometry of Scanning Ion Conductance Microscope Pipets. Anal. Chem. 2012, 84, 8980-8984.

(16) Knowles, M. R.; Robinson, J. M.; Wood, R. E.; Pue, C. A.; Mentz, W. M.; Wager, G. C.; Gatzy, J. T.; Boucher, R. C. Ion Composition of Airway Surface Liquid of Patients with Cystic Fibrosis as Compared with Normal and Disease-Control Subjects [Published Erratum Appears in J Clin Invest 1998 Jan 1;101(1):285]. J. Clin. Invest. 1997, 100, 2588-2595.

(17) Hull, J.; Skinner, W.; Robertson, C.; Phelan, P. Elemental Content of Airway Surface Liquid from Infants with Cystic Fibrosis. Am. J. Respir. Crit. Care Med. 1998, 157, 10-14.

(18) Rheinlaender, J.; Schäffer, T. E. Image Formation, Resolution, and Height Measurement in Scanning Ion Conductance Microscopy. J. Appl. Phys. 2009, 105, 094905.

(19) Del Linz, S.; Willman, E.; Caldwell, M.; Klenerman, D.; Fernández, A.; Moss, G. Contact-Free Scanning and Imaging with the Scanning Ion Conductance Microscope. Anal. Chem. 2014, 86, 23532360.

(20) Chen, C.-C.; Zhou, Y.; Morris, C. A.; Hou, J.; Baker, L. A. Scanning Ion Conductance Microscopy Measurement of Paracellular Channel Conductance in Tight Junctions. Anal. Chem. 2013, 85, 3621-3628.

(21) Paradiso, A. M.; Ribeiro, C. M. P.; Boucher, R. C. Polarized Signaling via Purinoceptors in Normal and Cystic Fibrosis Airway Epithelia. J. Gen. Physiol. 2000, 117, 53-68.

(22) Edwards, M. A.; Williams, C. G.; Whitworth, A. L.; Unwin, P. R. Scanning Ion Conductance Microscopy: A Model for Experimentally Realistic Conditions and Image Interpretation. Anal. Chem. 2009, 81, 4482-4492.

(23) Cole, R. W.; Jinadasa, T.; Brown, C. M. Measuring and Interpreting Point Spread Functions to Determine Confocal Microscope Resolution and Ensure Quality Control. Nat. Protoc. 2011, 6, 1929-1941.

(24) Liu, L.; Gardecki, J. A.; Nadkarni, S. K.; Toussaint, J. D.; Yagi, Y.; Bouma, B. E.; Tearney, G. J. Imaging the Subcellular Structure of Human Coronary Atherosclerosis Using Micro-Optical Coherence Tomography. Nat. Med. 2011, 17, 1010-1014.

(25) Tarran, R.; Trout, L.; Donaldson, S. H.; Boucher, R. C. Soluble Mediators, Not Cilia, Determine Airway Surface Liquid Volume in Normal and Cystic Fibrosis Superficial Airway Epithelia. J. Gen. Physiol. 2006, 127, 591-604.

(26) Tarran, R.; Button, B.; Picher, M.; Paradiso, A. M.; Ribeiro, C. M.; Lazarowski, E. R.; Zhang, L.; Collins, P. L.; Pickles, R. J.; Fredberg, J. J.; et al. Normal and Cystic Fibrosis Airway Surface Liquid Homeostasis. The Effects of Phasic Shear Stress and Viral Infections. J. Biol. Chem. 2005, 280, 35751-35759.

(27) Smith, C. M.; Djakow, J.; Free, R. C.; Djakow, P.; Lonnen, R.; Williams, G.; Pohunek, P.; Hirst, R. A.; Easton, A. J.; Andrew, P. W.; et al. CiliaFA: A Research Tool for Automated, High-Throughput 
Measurement of Ciliary Beat Frequency Using Freely Available Software. Cilia 2012, 1, 14.

(28) Chen, J. J.; Lemieux, B. T.; Wong, B. J. F. A Low-Cost Method of Ciliary Beat Frequency Measurement Using IPhone and MATLAB: Rabbit Study. Otolaryngol. Head Neck Surg. 2016, 155, 252-256.

(29) Feng, W.; Garrett, H.; Speert, D. P.; King, M. Am. J. Respir. Crit. Care Med. 1998, 157, 710-714.

(30) Pezzulo, A. A.; Tang, X. X.; Hoegger, M. J.; Abou Alaiwa, M. H.; Ramachandran, S.; Moninger, T. O.; Karp, P. H.; WohlfordLenane, C. L.; Haagsman, H. P.; van Eijk, M.; et al. Reduced Airway Surface PH Impairs Bacterial Killing in the Porcine Cystic Fibrosis Lung. Nature 2012, 487, 109-113.

(31) Itani, O. A.; Chen, J.-H.; Karp, P. H.; Ernst, S.; Keshavjee, S.; Parekh, K.; Klesney-Tait, J.; Zabner, J.; Welsh, M. J. Human Cystic Fibrosis Airway Epithelia Have Reduced Cl- Conductance but Not Increased Na+ Conductance. Proc. Natl. Acad. Sci. U.S.A. 2011, 108, 10260-10265.

(32) Schultz, A.; Puvvadi, R.; Borisov, S. M.; Shaw, N. C.; Klimant, I.; Berry, L. J.; Montgomery, S. T.; Nguyen, T.; Kreda, S. M.; Kicic, A.; et al. Airway Surface Liquid PH Is Not Acidic in Children with Cystic Fibrosis. Nat. Commun. 2017, 8, 1-8.

(33) Morris, C. A.; Chen, C.-C.; Ito, T.; Baker, L. A. Local PH Measurement with Scanning Ion Conductance Microscopy. J. Electrochem. Soc. 2013, 160, H430-H435.

(34) Nadappuram, B. P.; McKelvey, K.; Al Botros, R.; Colburn, A. W.; Unwin, P. R. Fabrication and Characterization of Dual Function Nanoscale PH-Scanning Ion Conductance Microscopy (SICM) Probes for High Resolution PH Mapping. Anal. Chem. 2013, 85, 8070-8074.

(35) Zhuang, J.; Jiao, Y.; Li, Z.; Lang, J.; Li, F. A Continuous Control Mode with Improved Imaging Rate for Scanning Ion Conductance Microscope (SICM). Ultramicroscopy 2018, 190, 66-76.

(36) Leopold, P. L.; O’Mahony, M. J.; Julie Lian, X.; Tilley, A. E.; Harvey, B. G.; Crystal, R. G. Smoking Is Associated with Shortened Airway Cilia. PLoS One 2009, 4, e8157.

(37) Li, Y. Y.; Li, C. W.; Chao, S. S.; Yu, F. G.; Yu, X. M.; Liu, J.; Yan, Y.; Shen, L.; Gordon, W.; Shi, L.; et al. Impairment of Cilia Architecture and Ciliogenesis in Hyperplastic Nasal Epithelium from Nasal Polyps. J. Allergy Clin. Immunol. 2014, 134, 1282-1292.

(38) Shah, A. S.; Farmen, S. L.; Moninger, T. O.; Businga, T. R.; Andrews, M. P.; Bugge, K.; Searby, C. C.; Nishimura, D.; Brogden, K. A.; Kline, J. N.; et al. Loss of Bardet-Biedl Syndrome Proteins Alters the Morphology and Function of Motile Cilia in Airway Epithelia. Proc. Natl. Acad. Sci. U.S.A. 2008, 105, 3380-3385.

(39) Serafini, S. M.; Michaelson, E. D. Length and Distribution of Cilia in Human and Canine Airways. Bull Eur Physiopathol Respir 1977, 13, 551-559.

(40) Pablo, J. L.; DeCaen, P. G.; Clapham, D. E. Progress in Ciliary Ion Channel Physiology. J. Gen. Physiol. 2016, 149, 37-47.

(41) Nakajima, M.; Mizutani, Y.; Iwata, F.; Ushiki, T. Scanning Ion Conductance Microscopy for Visualizing the Three-Dimensional Surface Topography of Cells and Tissues. Semin. Cell Dev. Biol. 2018, $73,125-131$.

(42) Zhou, Y.; Saito, M.; Miyamoto, T.; Novak, P.; Shevchuk, A. I.; Korchev, Y. E.; Fukuma, T.; Takahashi, Y. Nanoscale Imaging of Primary Cilia with Scanning Ion Conductance Microscopy. Anal. Chem. 2018, 90, 2891-2895.

(43) Rahmoune, H.; Shephard, K. L. State of Airway Surface Liquid on Guinea Pig Trachea. J. Appl. Physiol. 1995, 78, 2020-2024.

(44) Harvey, P. R.; Tarran, R.; Garoff, S.; Myerburg, M. M. Measurement of the Airway Surface Liquid Volume with Simple Light Refraction Microscopy. Am. J. Respir. Cell Mol. Biol. 2011, 45, 592599.

(45) Thiagarajah, J. R.; Song, Y.; Derichs, N.; Verkman, A. S. Airway Surface Liquid Depth Imaged by Surface Laser Reflectance Microscopy. J. Gen. Physiol. 2010, 136, 353-362.

(46) Neubauer, D.; Korbmacher, J.; Frick, M.; Kiss, J.; Timmler, M.; Dietl, P.; Wittekindt, O. H.; Mizaikoff, B. Deuterium Oxide Dilution:
A Novel Method to Study Apical Water Layers and Transepithelial Water Transport. Anal. Chem. 2013, 85, 4247-4250.

(47) Jayaraman, S.; Song, Y.; Vetrivel, L.; Shankar, L.; Verkman, A. S. Noninvasive in Vivo Fluorescence Measurement of Airway Surface Liquid Depth, Salt Concentration, and PH. J. Clin. Invest. 2001, 107, 317-324.

(48) Birket, S. E.; Chu, K. K.; Liu, L.; Houser, G. H.; Diephuis, B. J.; Wilsterman, E. J.; Dierksen, G.; Mazur, M.; Shastry, S.; Li, Y.; et al. A Functional Anatomic Defect of the Cystic Fibrosis Airway. Am. J. Respir. Crit. Care Med. 2014, 190, 421-432.

(49) Shevchuk, A. I.; Gorelik, J.; Harding, S. E.; Lab, M. J.; Klenerman, D.; Korchev, Y. E. Simultaneous Measurement of Ca2+ and Cellular Dynamics: Combined Scanning Ion Conductance and Optical Microscopy to Study Contracting Cardiac Myocytes. Biophys. J. 2001, 81, 1759-1764.

(50) Takahashi, Y.; Shevchuk, A. I.; Novak, P.; Murakami, Y.; Shiku, H.; Korchev, Y. E.; Matsue, T. Simultaneous Noncontact Topography and Electrochemical Imaging by SECM/SICM Featuring Ion Current Feedback Regulation. J. Am. Chem. Soc. 2010, 132, 10118-10126.

(51) Elam, J. W.; Pellin, M. J.; Comstock, D. J.; Elam, J. W.; Pellin, M. J.; Hersam, M. C. Integrated Ultramicroelectrode-Nanopipet Probe for Concurrent Scanning Electrochemical Microscopy and Scanning Ion Conductance. 2010, 82 (July 2016), 1270-1276. https://doi.org/ DOI: 10.1021/ac902224q.

(52) Page, A.; Kang, M.; Armitstead, A.; Perry, D.; Unwin, P. R. Quantitative Visualization of Molecular Delivery and Uptake at Living Cells with Self-Referencing Scanning Ion Conductance Microscopy (SICM) - Scanning Electrochemical Microscopy (SECM). Anal. Chem. 2017, 89, 3021-3028. 